\title{
META-ANÁLISIS DE LA CALIDAD DE LOS SERVICIOS MÉDICOS SEGÚN LAS DIMENSIONES DEL SERVQUAL
}

Recibido: junio del 2018

Aceptado: febrero del 2019

Leidy Alexandra Lezcano Tobón ${ }^{1}$, Jaiberth Antonio Cardona-Arias ${ }^{2}$

\section{Resumen}

Introducción: La calidad de los servicios de salud es determinante para minimizar riesgos y maximizar la satisfacción de los pacientes. El Servqual es una escala con buena validez y reproducibilidad para medir la calidad. Objetivo: Meta-analizar la calidad de los servicios médicos según las dimensiones del Servqual. Métodos: Revisión sistemática de la literatura científica relacionada con Servqual en MedLine-PubMed, ScienceDirect, Lilacs, Redalyc y Scielo. Se aplicó la guía Prisma para la selección de estudios y Strobe para evaluar la calidad editorial y metodológica. El análisis se realizó con Galbraith, Dersimonian Laird's, Begg, Funnel y Forest Plot, para cada dimensión de calidad. Se realizó una meta-regresión según el tipo de servicio evaluado, institución y calidad metodológica. Resultados: Las áreas más estudiadas son consulta externa y hospitalización. El resultado global evidenció buena calidad con una diferencia de medias de 0,93 (IC de $95 \%=0,53-1,33$ ); en la dimensión de tangibilidad fue de 1,39 (IC de $95 \%=0,84-1,94$ ); en confiabilidad fue de 1,16 (IC de $95 \%=0,64-1,67$ ); en capacidad de respuesta fue de 1,03 (IC de $95 \%=0,50-1,57$ ), en seguridad fue de $0,91$ (IC de $95 \%=0,49-1,34)$ y en empatía fue de $0,94(0,40-1,49)$. El mejor perfil de calidad se halló en consulta externa y en las instituciones públicas. Conclusión: La investigación sobre evaluación de la calidad en salud, según las dimensiones del Servqual, resulta exigua en América. Se evidenció un buen perfil de calidad en los servicios médicos sistematizados, al identificar los de mejor desempeño e información relevante para mejorar los esfuerzos por la evaluación de la calidad como un desenlace clave en la atención en salud.

\footnotetext{
${ }^{1}$ Médica, M.Sc. de Administración en Salud. Universidad Cooperativa de Colombia.

${ }^{2}$ MyB, M.Sc. de Epidemiología, M.Sc. de Economía Aplicada. Candidato a Doctor en Salud Pública, Universidad de Antioquia.
} 


\section{META-ANALYSIS OF QUALITY OF MEDICAL SERVICES ACCORDING TO SERVQUAL}

Leidy Alexandra Lezcano Tobón ${ }^{1}$, Jaiberth Antonio Cardona-Arias ${ }^{2}$

\section{Abstract}

Introduction: The quality of health services is a determining factor to minimize any risk and increase patient satisfaction. Servqual is a scale with good validity and reproducibility to rate quality. Objective: To meta-analyze the quality of medical services according to the dimensions of Servqual. Method: Systematic revision of scientific literature related to Servqual in Medline-PubMed, ScienceDirect, Lilacs, Redalyc and Scielo. The guide prisma was used to select studies, and the guide Strobe was used to evaluate editorial and methodological quality. The analysis was made with Galbraith, Dersimonian Laird's, Begg, Funnel and Forest Plot for each dimension of quality. We carried out a meta regression according to the type of evaluated service, institution and methodological quality. Results: The most studied areas are consultation and hospitalization. A global result showed good quality with a mean difference of 0.93 (CI of $95 \%=0,53-1,33$ ); in tangibility, it was 1.39 (IC of $95 \%=0.84-1.94$ ), in reliability it was 1.16 (CI of $95 \%=0.64-1.67)$, in responsiveness, it was $1.03(\mathrm{CI}$ of $95 \%=0,50-$ $1,57)$, in safety it was 0.91 (CI of $95 \%=0.490-1,57)$, in safety it was 0.91 (CI of $95 \%=0.49-1.34)$ and in empathy it was $0.94(0.40-1.49)$. The best quality profile was found in consultation and in public institutions. Conclusion: research about evaluation of quality in health, according to the dimensions of Servqual, results meager in America. There was a good profile in quality of systematic services, since they have the best performance and most relevant information to improve the efforts of evaluation of quality as a key ending of health assistance. 


\title{
META-ANÁLISE DA QUALIDADE DOS SERVIÇOS MÉDICOS SEGUNDO AS DIMENSÕES DO SERVQUAL
}

\author{
Leidy Alexandra Lezcano Tobón ${ }^{1}$, Jaiberth Antonio Cardona-Arias ${ }^{2}$
}

\section{Resumo}

Introdução: a qualidade dos serviços de saúde é determinante para minimizar riscos e maximizar a satisfação dos pacientes. O ServQual é uma escala com boa validade e reprodutibilidade para medir a qualidade. Objetivo: meta-analisar a qualidade dos serviços médicos segundo as dimensões do ServQual. Métodos: revisão sistemática da literatura científica relacionada com o ServQual nas bases de dados: MedLine-PubMed, ScienceDirect, Lilacs, Redalyc e SciELO. Foi aplicado o guia Prisma para selecionar estudos e o Strobe para avaliar a qualidade editorial e metodológica. A análise foi realizada com Galbraith, Dersimonian Laird's, Begg, Funnel e Forest Plot, para cada dimensão de qualidade. Foi realizada metarregressão segundo o tipo de serviço avaliado, a instituição e a qualidade metodológica. Resultados: as áreas mais estudadas são consulta e internação. O resultado global evidenciou boa qualidade com uma diferença de médias de 0,93 (IC de $95 \%=0,53$ - 1,33); na dimensão de tangibilidade, foi de 1,39 (IC de $95 \%=0,84-1,94$ ); na de confiabilidade, foi de 1,16 (IC de $95 \%=$ 0,64 - 1,67); na de capacidade de resposta, foi de 1,03 (IC de $95 \%=$ 0,50 - 1,57); na de segurança, foi de 0,91 (IC de $95 \%=0,49-1,34$ ) e, na de empatia, foi de $0,94(0,40$ - 1,49). O melhor perfil de qualidade foi verificado em consulta e nas instituições públicas. Conclusão: esta pesquisa sobre avaliação da qualidade em saúde, segundo as dimensões do ServQual, é escassa na América. Constatou-se um bom perfil de qualidade nos serviços médicos sistematizados, ao identificar os de melhor desempenho; além disso, este estudo contribui com informação relevante para melhorar os esforços para avaliar a qualidade como um aspecto fundamental na atenção em saúde. 


\section{Introducción}

Los avances tecno-científicos en medicina han complejizado la prestación de los servicios de salud, al demandar altos estándares de calidad que minimicen los riesgos y maximicen la satisfacción de los usuarios; esto reviste mayor importancia al considerar los impactos económicos, de discapacidad, morbilidad o mortalidad asociados con el cuidado en salud (1). En este sentido, se estima que entre 44.000 y 98.000 estadounidenses mueren cada año por errores médicos, cifras que superan las muertes por accidentes automovilísticos, cáncer de mama o SIDA; al tiempo que los costos por manejo de eventos adversos prevenibles representan más de la mitad del presupuesto destinado a atención médica (2). Algunos estudios han referido 7.000 muertes anuales por errores en la medicación (3), otros reportan 0,8 errores de medicación por cada 100 admisiones (4) y una investigación del Colegio Estadounidense de Cirujanos encontró que el 8,9\% de los cirujanos refirieron errores médicos importantes en los últimos tres meses (5).

Los servicios de salud inseguros generan insatisfacción del paciente, riesgos legales, mayores índices de morbilidad y mortalidad, mayor costo, menor productividad, pérdida de confianza, malestar físico y psicológico, ausencias escolares y laborales, entre otros aspectos que evidencian la relevancia de disponer de servicios de salud con énfasis

en la seguridad del paciente y de alta calidad $(1,2)$.
En este orden de ideas, se disponen de múltiples acepciones de calidad del servicio, aunque la mayoría evidencia la centralidad de la perspectiva del usuario (6). Grönroos (1984) manifiesta que los servicios requieren una activa interacción entre el comprador y el proveedor, por lo que la calidad percibida implica el juicio subjetivo del cliente sobre el servicio que recibe (7).

Zeithaml entiende la calidad percibida como el juicio de un consumidor acerca de la excelencia total o superioridad de un producto o servicio (8). Parasumaran, Zeithaml y Berry (1985) definen la calidad como el resultado de la comparación de los deseos del consumidor y sus percepciones frente al servicio recibido (9), en los atributos de tangibilidad, fiabilidad, capacidad de respuesta, seguridad y empatía, las cuales se asumen en la escala SERVQUAL (Service Quality) $(9,10)$.

En particular, la calidad de los servicios de salud, según el Instituto de Medicina (IOM) en 1990, es "el grado en que los servicios de salud para las personas y las poblaciones aumentan la probabilidad de los resultados de salud deseados y son coherentes con el conocimiento profesional actual" (11). Donabedian (1988) indica que "calidad de la atención es aquella que se espera que pueda proporcionar al usuario el máximo y más completo bienestar después de valorar el balance de ganancias y pérdidas que pueden acompañar el proceso en todas sus partes" (12). Estas definiciones resaltan tres componentes: estructura, proceso y resultado, el primero se refiere a los recursos utilizados para 
la adecuada prestación del servicio de salud, el proceso subsume la forma de prestación técnica del servicio y el resultado es el efecto que tiene la atención médica sobre la salud y el bienestar del paciente (13).

En este sentido, una revisión de la literatura sobre la medición de la percepción de la calidad de los servicios de salud indicó que, en el ámbito mundial, predomina la perspectiva del marketing con aplicación del Servqual (14). Estudios previos han evidenciado una eleva heterogeneidad en la aplicación de esta escala en centros de atención primaria (15), servicios de consulta externa (1619), hospitalización (20-22), urgencias (23), cirugía $(23,24)$, Unidad de Cuidados intensivos (25) o ayudas diagnósticas (23), con pocas aplicaciones en el contexto latinoamericano $(16,17,24)$.

En adición a lo anterior, también se evidencian diferencias relevantes en los perfiles de calidad hallados en estudios previos; así, en el estudio de Regaira se halló una diferencia global de 0,33 entre expectativa y percepción (en una escala de 0 a 5) en Unidad de Cuidados Intensivos (25), en la investigación de Borges en servicios de cirugía cardiaca fue 0,1 (24); en el de Kalaja 0,42 en servicios de hospitalización (22); mientras que otros han reportado promedio negativos como Ghahramanian con -2,93 en servicios quirúrgicos (26); Mohebifar -2,18 en hospitalización (27) y Rivera $-1,77$ en consulta externa (17). Igualmente, se ha encontrado heterogeneidad en los potenciales factores asociados con los puntajes de las dimensiones del Servqual, como el servi- cio evaluado y la tipología del hospital (público o privado) $(23,28)$.

Lo expuesto pone de manifiesto la elevada heterogeneidad de usos del Servqual, así como los resultados de los perfiles hallados en sus diferentes dimensiones. Si bien existen algunas revisiones previas del tema, estas se restringen a algún país (29) o a una limitada ventana de tiempo (30), lo que evidencia la necesidad de ampliar el análisis de los resultados del Servqual como instrumento de valoración de la calidad de los servicios médicos en el mundo, con el fin de aumentar la validez externa de las conclusiones, consolidar hipótesis sobre las áreas clínicas evaluadas y los servicios hospitalarios con mejor calidad percibida; así como otras recomendaciones que permitan orientar la toma de decisiones en la gestión de servicios de salud, orientar acciones a la garantía de la calidad en salud, mejorar el uso de recursos $y$, en el marco de la medicina basada en la evidencia, la inclusión de la perspectivas del paciente o desenlaces centrados en sus expectativas.

El objetivo de este estudio fue meta-analizar la percepción de la calidad de los servicios médicos según las dimensiones del Servqual.

\section{Métodos}

Tipo de estudio: revisión sistemática de la literatura con meta-análisis.

\section{Descripción de la escala Servqual}

Es una escala con buena validez (cargas factorial mayores a 0,30$)$ y confiabili- 
dad (Alfa de Cronbach mayor a 0,70) que permiten valorar la calidad de los servicios a través de la medición de las expectativas (previo a la atención) y percepciones (inmediatamente finaliza la atención) de los usuarios a partir de 44 ítems, 22 de expectativas y 22 de percepción, agrupados en cinco dimensiones:

1. Elementos tangibles (tangibilidad) que incluyen la evaluación de las instalaciones físicas, equipamiento $\mathrm{y}$ apariencia del personal.

2. Fiabilidad que da cuenta de la capacidad de realizar el servicio prometido de manera confiable y precisa.

3. Capacidad de respuesta que mide la voluntad de ayudar a los pacientes y brindar un servicio rápido.

4. Seguridad o garantía que evalúa competencia, cortesía y seguridad.

5. Empatía que incluye ítems relacionados con atención solidaria e individualizada.

Esta estructura puede adaptarse a las necesidades de diferentes organizaciones y al finalizar la calificación de la calidad se da en términos de la diferencia entre las expectativas y la percepción por cada dimensión, también se puede estimar un puntaje general con todos los ítems $(10,31)$. Para los servicios de salud, esta escala fue adatada y validada en 1992 por Babakus (32) y se dispone de varios estudios de adaptación a diferentes idiomas (33), países (34) y servicios médi-

138 cos (35). El puntaje por cada dimensión o el global corresponde a un promedio, donde el valor nulo es cero (que refleja igualdad en la expectativa y la percepción); los valores negativos reflejan problemas de calidad, ya que corresponden a un mayor promedio en la expectativa, mientras que los valores positivos indican excelente calidad dado que corresponde a mayores puntajes en la percepción en comparación con la expectativa.

Pregunta PICO (Población, Intervención, Comparador, Resultado) (Outcome)

Población: pacientes o familiares que hayan sido atendidos en servicios médicos hospitalarios.

Intervención: medición de la expectativa de la calidad en diferentes servicios hospitalarios, según las dimensiones del Servqual.

Comparador: medición de la percepción de la calidad del mismo servicio hospitalario según Servqual.

Resultado: diferencia entre la expectativa y la percepción de calidad en cada dimensión de la escala y en el resultado global.

Protocolo de búsqueda y selección de los estudios según las fases de la guía Prisma (Preferred Reporting Items for Systematic Reviews and Meta-Analyse (36).

Identificación: se realizó una búsqueda de la literatura científica con un único término de búsqueda Servqual en las bases de datos MedLine-PubMed, ScienceDirect, Lilacs, Redalyc y Scielo. 
Tamización: los criterios de inclusión fueron:

1. Investigaciones con término de búsqueda en el título o el resumen.

2. Artículos originales.

3. El eje central del artículo fuese la aplicación del Servqual en servicios hospitalarios.

4. Estudios observacionales, transversales y longitudinales.

5. Que tuviera los resultados de la expectativa y la percepción para determinar la diferencia en las cinco dimensiones de la escala.

Algunas sintaxis de búsqueda fueron las siguientes SERVQUAL[Title/Abstract], Title, abstract, keywords: SERVQUAL, (ti:((ab:(SERVQUAL)))). No se aplicaron restricciones temporales de manera retrospectiva, la última actualización del protocolo de búsqueda se realizó en mayo del 2018.

Elección: como criterios de exclusión se definieron:

1. Estudios de validación del Servqual o en los que este fue empleado para validar otros instrumentos.

2. Publicaciones que no reportaron los puntajes de cada dimensión de la calidad.

3. Artículos con bajo número de pacientes (menos de 10).
4. Estudios no disponibles (e incluidos en el meta-análisis de Irán) en las bases de datos (solo reportan el título) pese a la solicitud a los autores.

Inclusión: los estudios que cumplieron el protocolo se analizaron mediante síntesis cualitativa de las variables: título, autores, año de publicación, país, número de sujetos de estudio, servicios evaluados y factores asociados con la calidad; en adición, se realizó una síntesis cuantitativa (meta-análisis) de los puntajes de la expectativa y la percepción de calidad de las dimensiones de Servqual.

Análisis de reproducibilidad y evaluación de la calidad metodológica: se garantizó la reproducibilidad de la selección de los estudios y la extracción de la información por medio del diligenciamiento del protocolo Prisma y la extracción de variables en un archivo plano de Excel, por parte de dos investigadores de manera independiente. La calidad editorial y metodológica de los estudios se realizó con la guía Strobe (Strengthening the Reporting of Observational studies in Epidemiology) (37).

Análisis estadístico: la descripción se basó en frecuencias. Se realizó metanálisis para la diferencia de medias (puntaje expectativa - puntaje percepción) en cada dimensión del Servqual, previa estandarización de los puntajes en una escala de cero a cinco, dado que algunos estudios usaron escalas con diferente amplitud de rango. La diferencia de medias tomó valores entre - 5 (peor calidad percibida, dado que la expectativa superó en el valor máximo a la percepción) 
y 5 (mejor calidad posible, dado que la percepción superó en el valor máximo a la expectativa).

Se hizo análisis de heterogeneidad con el método gráfico de Galbraith y los métodos analíticos de Dersimonian Laird's (Estadístico Q Ji-cuadrado) e I2; se estimó el sesgo de publicación con el estadístico de Begg (estadístico Z) y el Funnel Plot, el análisis de sensibilidad con el gráfico de influencia y el metanálisis global con el Forest Plot de cada dimensión de calidad. Se realizó meta-regresión según el tipo de servicio evaluado (consulta y hospitalización, dado que los demás servicios se evaluaron en menos de tres estudios), el tipo de institución (público o privada) y la calidad metodológica de los estudios (agrupada en buenas para aquellos que cumplieron entre 80 y $90 \%$ de los criterios Strobe y excelente a partir del 90\%).

\section{Resultados}

En la búsqueda inicial se identificaron 2.612 publicaciones de las cuales se tamizaron 497 que incluyeron el nombre de la escala en título o resumen, con base en la aplicación de criterios de inclusión y exclusión se meta-analizaron 14 publicaciones (figura 1 ).
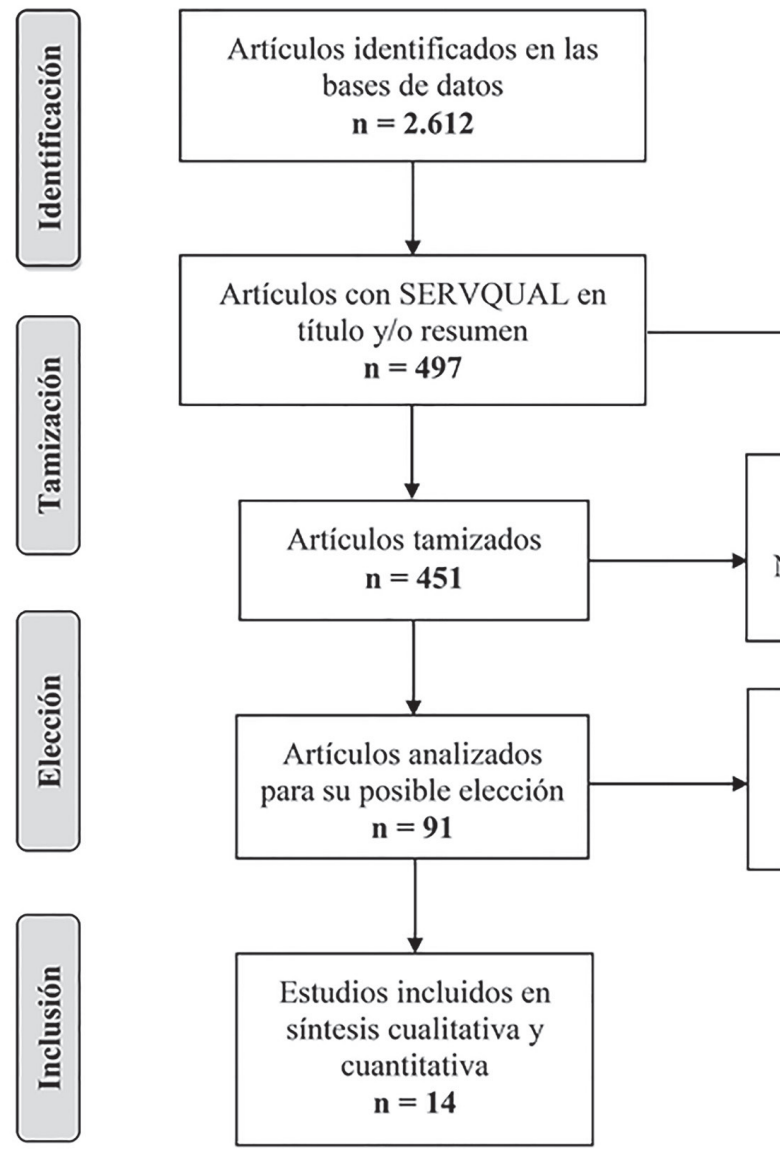

Artículos excluidos Duplicados $\mathrm{n}=46$

No es en salud $\mathbf{n}=\mathbf{2 2 8}$

No es original $\mathbf{n}=\mathbf{1 8}$

No se realiza en hospitales $\mathbf{n}=\mathbf{5 3}$

No aplica SERVQUAL $\mathbf{n}=\mathbf{6 1}$

Validación Adaptación $\mathbf{n}=\mathbf{8}$

No da puntaje de calidad $\mathbf{n}=\mathbf{3 0}$

No disponible e incluidos en metanálisis de Irán $\mathbf{n}=\mathbf{3 9}$

Figura 1. Flujograma de búsqueda y selección de los estudios Fuente: elaboración propia. 
Los estudios se publicaron entre $2009 \mathrm{y}$ 2017, la mayor proporción en Asia, con un total de 4.682 individuos analizados, quienes evaluaron principalmente consulta externa, hospitalización y en menor frecuencia cirugía, urgencias, UCI,
UCE, atención primaria, ginecología y ayudas diagnósticas (tabla 1). Cinco estudios se desarrollaron en instituciones públicas, cuatro en privadas y en los demás artículos no es explicitó el tipo de institución de salud.

Tabla 1. Descripción de los estudios según año, país, muestra y servicio evaluado

\begin{tabular}{|c|c|c|c|c|}
\hline Autor & Año & País & $\mathrm{N}$ & Servicios \\
\hline Mohd (21) & 2009 & Malasia & 191 & Hospitalización \\
\hline Borges (24) & 2010 & Brasil & 82 & Cirugía \\
\hline Huang (38) & 2010 & Taiwán & $685 a$ & Consulta externa \\
\hline Regaira (25) & 2010 & España & 86 & UCl y UCE \\
\hline Chakravarty (19) & 2011 & India & 50 & Consulta externa \\
\hline Lorin (39) & 2013 & Rumania & 183 & Ginecología \\
\hline Fernández (16) & 2014 & Perú & $470 b$ & Consulta externa \\
\hline Khamis (18) & 2014 & Tanzania & 422 & Consulta externa \\
\hline Papanikolaou (15) & 2014 & Grecia & 450 & $\begin{array}{c}\text { Centros de salud y atención } \\
\text { primaria }\end{array}$ \\
\hline Rivera (17) & 2015 & Perú & 328 & Consulta externa \\
\hline Al Fraihi (20) & 2016 & Arabia & 306 & Hospitalización \\
\hline Kalaja (22) & 2016 & Albania & 200 & Hospitalización \\
\hline Manulik (28) & 2016 & Polonia & 412 & Clínicas de alergias \\
\hline Fatima (23) & 2017 & Pakistán & $817 b$ & $\begin{array}{c}\text { Urgencias, Cirugía y Ayudas } \\
\text { diagnósticas }\end{array}$ \\
\hline
\end{tabular}

a Paciente, familiares y personal de la institución. b Pacientes y familiares. 
Todos los estudios presentaron una buena calidad metodológica al cumplir $80 \%$ o más de los criterios de la guía Strobe; cuatro estudios cumplieron entre el $80 \mathrm{y}$ $90 \%$ de los ítems, en los demás el porcentaje de cumplimiento de los criterios de calidad fue mayor al $90 \%$; sin embargo, algunos ítems presentaron una baja proporción de aplicación como la declaración de la financiación, las limitaciones y los análisis adicionales de subgrupos (figura 2).

\begin{tabular}{lrr}
\hline Criterio STROBE & \% de Estudios que cumplen el criterio \\
\hline Título/ Resumen & $\mathbf{1 0 0}$ \\
Fundamentación & $\mathbf{1 0 0}$ \\
Objetivo & $\mathbf{1 0 0}$ \\
Diseño del estudio & $\mathbf{1 0 0}$ \\
Contexto & $\mathbf{1 0 0}$ \\
Participantes & $\mathbf{1 0 0}$ \\
Definición de variables & $\mathbf{1 0 0}$ \\
Fuente de datos & $\mathbf{1 0 0}$ \\
Control de sesgos & $\mathbf{1 0 0}$ \\
Tamaño de muestra & $\mathbf{1 0 0}$ \\
Variables cuantitativas & $\mathbf{1 0 0}$ \\
Análisis estadísticos & $\mathbf{1 0 0}$ \\
Resultados de los participantes & $\mathbf{1 0 0}$ \\
Resultados principales & $\mathbf{1 0 0}$ \\
Análisis adicionales & $\mathbf{6 4}$ \\
Discusión de resultados clave & $\mathbf{1 0 0}$ \\
Limitaciones & $\mathbf{5 7}$ \\
Interpretaciones & $\mathbf{1 0 0}$ \\
Discusión de la generalización & $\mathbf{7 9}$ \\
Financiación & $\mathbf{5 0}$ \\
\hline
\end{tabular}

Figura 2. Evaluación de la calidad metodológica de los estudios incluidos

Fuente: elaboración propia.

En los estudios meta-analizados se halló una alta heterogeneidad (figura 3A) con Dersimonian Laird's de 994 ( $\mathrm{p}=0,000)$ e I2 de $98 \%$, lo que evidenció la necesidad de realizar un meta-análisis de efectos aleatorios. No se halló sesgo de publicación (figura 3B) con prueba de Begg de $0,11(p=0,913)$ y en el análisis de sensibilidad ningún estudio presentó un mayor peso sobre la estimación global de calidad (figura 3C), lo que corroboró la pertinencia de la medida de resumen final, en la cual se halló una diferencia de medias de 0,93 (IC de $95 \%=0,53-1,33$ ) bajo un modelo de efectos aleatorios que indica una buena percepción de calidad de los servicios evaluados con el Servqual, ya que la media de la percepción superó el promedio hallado en la expectativa (figura 3D). 


\section{A. Gráfico de Galbraith}

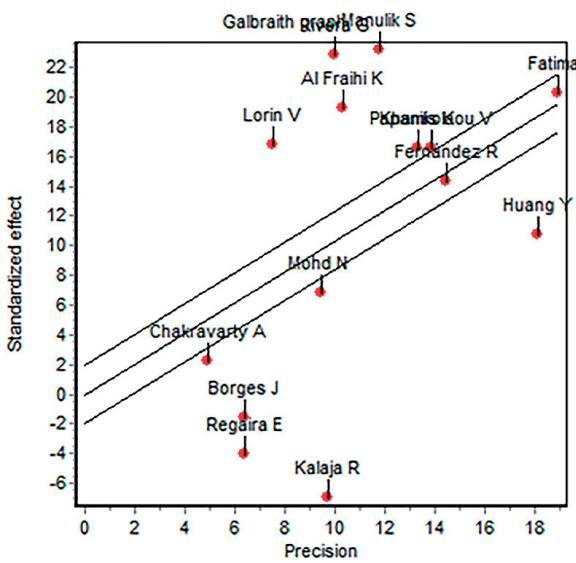

C. Gráfico de Influencia

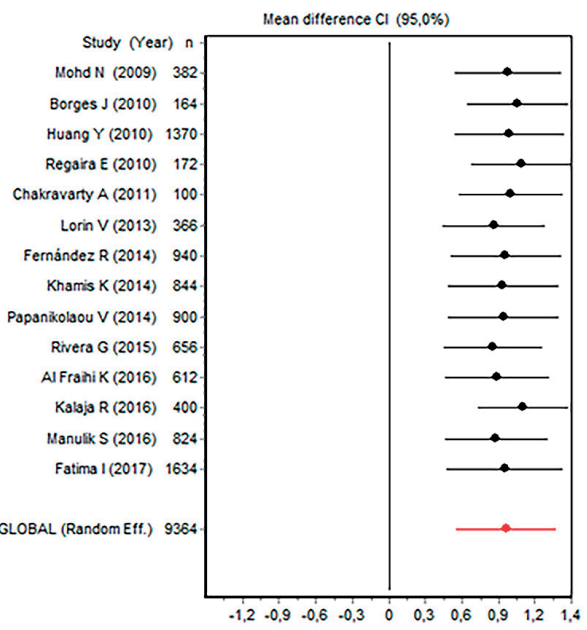

B. Funnel plot

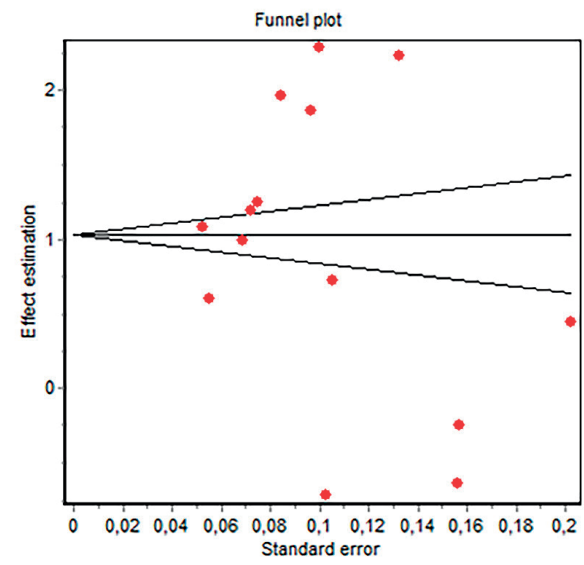

D. Forest Plot.

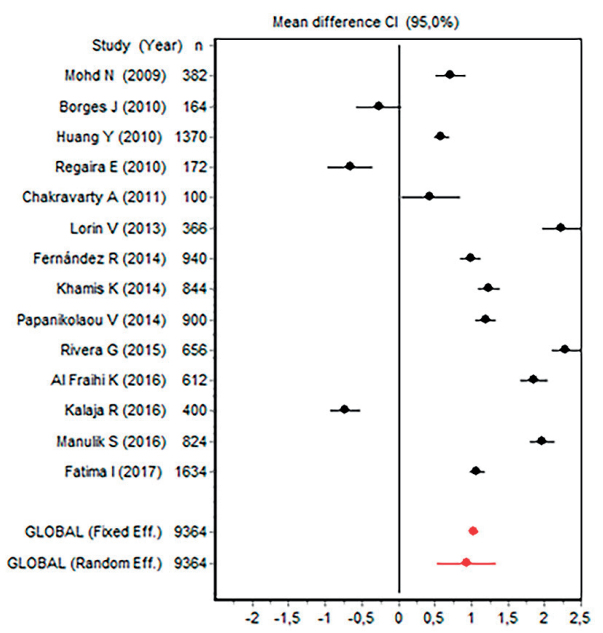

Figura 3. Meta-análisis del puntaje global de la percepción de la calidad de los servicios de salud (A. Gráfico de Glabraith, B. Funnel Plot, C. Gráfico de influencia y D. Forest Plot)

Fuente: elaboración propia.

En todas las dimensiones del Servqual se halló heterogeneidad según el método gráfico, el Dersimonian Laird's ( $\mathrm{p}<$ $0,05)$ e $\mathrm{I}^{2}(>50 \%)$, por lo que se estimaron medidas globales con el modelo de efectos aleatorios. En la dimensión de tangibilidad se halló una diferencia de medias de 1,39 (IC de $95 \%=0,84$ $1,94)$; en confiabilidad fue de 1,16 (IC de $95 \%=0,64-1,67)$; en capacidad de respuesta fue de 1,03 (IC de $95 \%=$ $0,50-1,57)$; en seguridad fue de 0,91 (IC de $95 \%=0,49-1,34$ ) y en empatía fue de $0,94(0,40$ - 1,49); lo que evidencia una buena calidad de la prestación de servicios de salud, sin diferencias estadísticas entre las dimensiones (figura 4). 

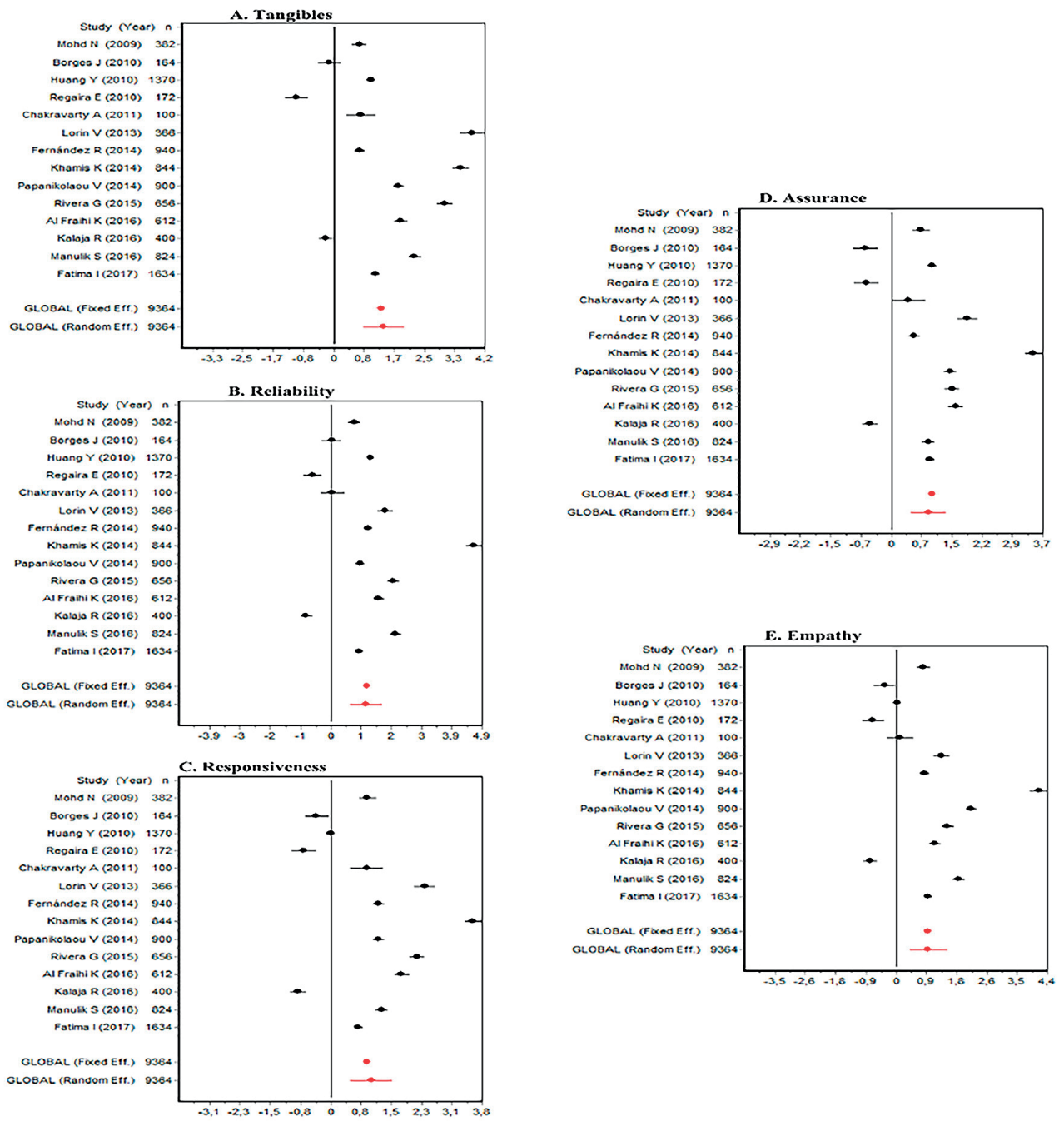

Figura 4. Forest Plot para las dimensiones del Servqual Fuente: elaboración propia.

En ninguna dimensión se halló sesgo de publicación y los análisis de sensibilidad evidenciaron que ningún estudio presentó mayor influencia sobre la medida combinada en comparación con los demás.

$\mathrm{Al}$ realizar las meta-regresiones del puntaje global de la calidad percibida se

144 hallaron cambios importantes según el tipo de servicio de salud, en la medida en que los estudios que evaluaron consulta externa presentaron una mejor calidad, ya que la percepción del servicio recibido superó la expectativa, mientras que en hospitalización no se hallaron diferencias estadísticas entre el puntaje de la expectativa y la percepción. De igual forma, los meta-análisis por tipo de institución evidenciaron diferencias positivas y estadísticamente significativas en las públicas y las privadas, sien- 
do mejor la percepción en las primeras. Finalmente, en los estudios de baja calidad metodológica no se hallaron diferencias estadísticas entre la expectativa y la percepción, mientras que en los de excelente calidad la percepción fue estadísticamente mayor a la expectativa (tabla 2).

Tabla 2. Análisis estratificado de la prevalencia de parasitismo intestinal según grupo etario y etnia

\begin{tabular}{|c|c|}
\hline & Servqual global (IC de $95 \%)$ a \\
\hline Todos los estudios & $0,93(0,53 ; 1,33)^{* *}$ \\
\hline \multicolumn{2}{|l|}{ Meta-regresiones } \\
\hline Consulta externa $\mathrm{N}=1.955$ & $1,12(0,57 ; 1,67)^{* *}$ \\
\hline Hospitalización N = 697 & $0,62(-0,87 ; 2,10)$ \\
\hline \multicolumn{2}{|l|}{ Por tipo de institución } \\
\hline Pública N = 1.725 & $0,98(0,91 ; 1,05)^{* *}$ \\
\hline Privada - mixta $N=1.506$ & $0,79(0,00 ; 1,57) *$ \\
\hline \multicolumn{2}{|l|}{ Por calidad metodológica } \\
\hline Buena $(80 \%-90 \%) \mathrm{N}=1.263$ & $0,91(-0,21 ; 2,02)$ \\
\hline Excelente (>90\%) N= 3.419 & $0,95(0,57 ; 1,33)^{* *}$ \\
\hline
\end{tabular}

a Diferencia de medias (expectativa - Percepción). ${ }^{*} p<0,05$. ${ }^{* *} p<0,01$.

Fuente: elaboración propia.

\section{Discusión}

Abordar el concepto de calidad en los servicios de salud implica necesariamente considerar las expectativas y las necesidades de los pacientes. En este sentido, el modelo de desconfirmación expuesto por Parasuraman, Zeithml y Berry ha sido ampliamente aceptado (40). Para estos autores, la calidad del servicio depende de la brecha entre la calidad esperada y la calidad percibida, donde un servicio es de calidad cuando la percepción es igual o superior a las expectativas, o sea, que la brecha es igual o mayor a cero $(9,40)$. Por su parte, el Servqual es la escala de medición estructurada en las cinco dimensiones de la calidad de los servicios propuestos que asume esta visión de la calidad centrada en la expectativa y la percepción del paciente (10).

En este estudio, en el resultado global del Servqual se halló una diferencia de medias de 0,93 bajo un modelo de efectos aleatorios, lo que indica una buena percepción de calidad de los servicios evaluados. Estos resultados son opuestos a los encontrados por Teshnizi en su meta-análisis sobre la calidad de los servicios de salud en Irán, en el cual se encontró una diferencia negativa $(-1,64)$ entre la percepción y las expectativas globales del servicio (29). Esto puede deberse al poco éxito que ha tenido la 
implementación de intervenciones para garantizar la calidad y las reformas del sistema de salud en Irán en los últimos años, explicado en parte por la amplia participación del estado iraní en la prestación de servicios y acreditación de instituciones, lo que no sucede en otros países del mundo (41-43).

Las dimensiones hacen referencia a los atributos del servicio que el paciente puede percibir y valorar, los cuales determinarán el juicio que este emita sobre la calidad del servicio (10). En este sentido, la dimensión de tangibilidad que hace referencia a elementos que pueden evaluarse objetivamente como infraestructura, dotación, presentación del personal y material de comunicación, implican mayor facilidad para su valoración (10), pese a ello, los resultados difieren entre estudios. Mientras que, en esta revisión, la tangibilidad fue satisfactoria con una diferencia de medias de 1,39 , en otro estudio fue insatisfactoria con una diferencia de medias entre $-0,31$ y $-0,05$. En este último caso, los autores identificaron cambios recientes de administración y trabajos de remodelación del hospital como limitaciones del estudio, las cuales pudieron inferir en los resultados, insinuando nuevas variables que pudiesen influenciar la percepción de este dominio de la calidad (44).

La atención médica se considera de alto nivel de credibilidad $(12,13)$, lo que le confiere gran relevancia a las dimensiones de confiabilidad, capacidad de respuesta, seguridad y empatía. La con-

146 fiabilidad hace referencia a la capacidad de realizar el servicio prometido de manera confiable y precisa (10) y fue la segunda dimensión mejor evaluada en nuestro estudio, con una diferencia de 1,16 , lo que contrasta con una revisión sistemática de Irán, donde la diferencia fue negativa de $(-1,15)(29)$. La confiabilidad está ligada a la estandarización de procesos y gestión de operaciones de salud (planear, controlar y mejorar) para optimizar los resultados y las experiencias del paciente, reflejado ello en puntualidad y en disminución de tiempos de espera y errores (45), lo que supone que Irán presenta una gestión operativa de servicios de salud deficiente, siendo esta una prioridad del gobierno actual (46).

Este estudio halló una diferencia de 1,03 en la dimensión de capacidad de respuesta, entendida como la voluntad de ayudar a los pacientes y proporcionar un servicio rápido y de 0,94 en la dimensión de empatía, la que hace referencia a la atención individual y cuidadosa que el hospital brinda a sus pacientes. En esta línea, un estudio halló una buena percepción sobre las dimensiones de confiabilidad y empatía en los usuarios de un programa salud de la familia (PSF) y describió el tiempo de residencia en el mismo territorio como un factor esencial para la creación de vínculos entre usuarios y profesionales del PSF, lo que redunda en la satisfacción de las expectativas de la población $\mathrm{y}$, por tanto, en una mejor calidad del servicio (47).

Las áreas con mayor aplicación de la escala fueron consulta externa y hospitalización. Esto puede explicarse, en parte, por la metodología propia de 
aplicación de la prueba y la necesidad de realizar la valoración de las expectativas previo a la atención, lo cual es logísticamente más complejo en servicios como urgencias, donde puede haber un menor tiempo de espera y menos disposición de participar en el estudio, por las condiciones de salud de los consultantes; sin embargo, es llamativo que de los estudios revisados en el meta-análisis reportan mejor calidad en servicios de alta complejidad, en UCI (25) y en cirugía cardiaca (24). El manejo de pacientes con condiciones de salud críticas requiere minimizar los riesgos de la atención y, por tanto, de un alto compromiso de las instituciones con la calidad y seguridad del paciente. Instituciones como la evaluada en el estudio de Regaira respaldan este compromiso con procesos de acreditación como la Joint Comission International (JCI) que podrían explicar el hallazgo expuesto (25).

Los estudios que evaluaron consulta externa presentaron una mejor calidad global (la percepción del servicio recibido superó la expectativa), mientras que en hospitalización no se hallaron resultados satisfactorios de calidad, dado que la expectativa no fue cumplida. Esto es coherente con los resultados del otro estudio que evalúa la calidad del servicio en los hospitales de nueve ciudades chinas, donde los visitantes de las instalaciones ambulatorias informaron una percepción más positiva que los visitantes de las instalaciones hospitalarias sobre los elementos tangibles y la confiabilidad (48). Las necesidades clínicas de consulta externa son más focales y, por lo tanto, también las expectativas del paciente. Hay menos necesidad de infraestructura, tecnología dura y menor tiempo de contacto con el personal, lo que favorece la percepción de los elementos tangibles. A su vez, el mayor control sobre el tiempo y la relación uno a uno en la atención redunda en mejor percepción de confiabilidad, así como en la capacidad de respuesta, seguridad y empatía.

Se evidenciaron diferencias positivas y estadísticamente significativas en el puntaje global de la calidad en las instituciones públicas, lo que puede atribuirse al hecho de que los pacientes de instituciones privadas tienen expectativas más altas (28). Además, en el hospital público de Durres, las mejoras en la calidad del servicio se reflejaron en una diferencia positiva entre las expectativas y las percepciones de los pacientes, explicada por buena percepción y bajas expectativas debido a experiencias previas poco satisfactorias (22).

Medir la percepción de la calidad del servicio partiendo del modelo de desconfirmación, en lugar de la medición exclusiva de la percepción, aporta una mayor capacidad diagnóstica para identificar áreas de deficiente calidad que requieran de intervenciones gerenciales al indicar la "distancia" entre lo esperado y lo percibido en los atributos del servicio, siendo imperativa la medición de las expectativas del paciente cuando se busca este fin (49).

También es importante indicar que los críticos del Servqual consideran ineficiente e innecesario medir las expectativas, dado que las personas tienden a 
tener muchas que raramente serán excedidas por las percepciones $(14,50)$. Los resultados de este estudio desvirtúan este argumento al mostrar que cuando un servicio es de calidad o mejora su calidad, la percepción sí puede superar las expectativas al reforzar la superioridad diagnóstica del Servqual frente al Servperf.

Finalmente, se hallaron deficiencias en los estudios en relación con el reporte de los potenciales factores asociados con la calidad de los servicios médicos, pese al reporte del sexo $(15,20,23,24)$, la edad $(15,20,24)$, la estancia hospitalaria (27), el nivel de escolaridad (15, 20, 23) y el número de atenciones previas (20) como factores asociados con la calidad en algunos estudios.

Entre las limitaciones del estudio se hallan las deficiencias en el reporte de factores asociados, el perfil de calidad de la atención médica por subgrupos de interés, entre otros aspectos que impidieron realizar otras meta-regresiones. Futuras investigaciones podrían orientarse a superar estas limitaciones y explorar asociaciones entre la calidad del servicio y las variables de las instituciones como su ubicación, tamaño, nivel de complejidad y acreditaciones de calidad. Pese a estas limitaciones, se evidencia que los sistemas sanitarios y particularmente un subsistema de atención médica presentan buena calidad desde la perspectiva de los pacientes, lo cual constituye un eje clave de la medicina basada en la evidencia, la atención humanizada, la medicina holística, entre otros campos que vindican la centralidad del paciente en la atención en salud.

\section{Conclusión}

La investigación sobre evaluación de la calidad en salud, según las dimensiones del Servqual, resulta exigua en América. Se evidenció un buen perfil de calidad en los servicios médicos sistematizados al identificar los de mejor desempeño, información relevante para mejorar los esfuerzos por la evaluación de la calidad como un desenlace clave en la atención en salud.

\section{Conflicto de interés}

Ninguno de los autores declara conflicto de intereses para la publicación del estudio.

\section{Financiación}

Recursos en especie de la Universidad Cooperativa de Colombia y de la Universidad de Antioquia. 


\section{Referencias bibliográficas}

1. Kapoor BP. Why quality in healthcare? Med J Armed Forces India. 2011;67(3):206-8.

2. Kohn LT, Corrigan JM, Donaldson MS. To err is human: building a safer health system. Annales francaises d'anesthesie et de reanimation. 2000;2:453-4.

3. Phillips D, Christenfiels N, Glynn L. Increase in US medication error deaths between 1983 and 1993. Lancet. 1998;351:643-4.

4. Choi I, Lee SM, Flynn L, Kim CM, Lee $S$, Kim NK, et al. Incidence and treatment costs attributable to medication errors in hospitalized patients. Res Soc Adm Pharm. 2016;12(3):428-37.

5. Shanafelt TD, Balch CM, Bechamps G, Russell T, Dyrbye L, Satele $D$, et al. Burnout and medical errors among American surgeons. Ann Surg. 2010;251(6):995-1000.

6. Radomir L, Plăiaş I, Nistor VC. A Review of the Service Quality Concept - Past, Present and Perspectives. Proc Int Conf Mark - from Inf to Decis. 2012;5:404-27.

7. Grönroos C. A service quality model and its marketing implications. Eur $\mathrm{J}$ Mark. 1984;18(4):36-44.

8. Zeithaml V. Consumer perceptions a means-end value: Quality and model synthesis of evidence. J Mark. 1988;52(julio):2-22.

9. Parasuraman A, Zeithaml V, Berry LL. A conceptual model of service quality and its implications for future research. $\mathrm{J}$ Mark. 1985;49(4):41-50.

10. Parasuraman A, Zeithaml VA, Berry LL. Servqual: A multiple-item scale for measuring consumer perceptions of service quality. J Retail. 1998;64(enero 2015):12-40.
11. Lohr KN. Medicare: A strategy for quality assurance. Washington D.C.: National Academy Press; 1990.

12. Donabedian A. Special article: The quality of care : How can it be assessed? JAMA. 1988;260(12):1743-8.

13. Donabedian A. Evaluating the quality of medical care. Milbank Q. 1966;83(4):166.

14. Losada M, Rodríguez A. Calidad del servicio de salud: una revisión a la literatura desde la perspectiva del marketing. Cuad Adm. 2007;20(34):237-58.

15. Papanikolaou V, Zygiaris S. Service quality perceptions in primary health care centres in Greece. Heal Expect. 2014;17(2):197-207.

16. Fernández R. Calidad de la atención y grado de satisfacción del paciente cardíaco transferido de provincia a la consulta externa de cardiología del INCOR 2009 [Tesis de maestría; impreso]. [Lima]: Universidad Nacional Mayor de San Marcos; 2014.

17. Rivera G. Satisfacción de los usuarios a través de sus percepciones y expectativas en la atención médica. Diagnóstico (Perú). 2015;54:5-9.

18. Khamis K, Njau B. Patients' level of satisfaction on quality of health care at Mwananyamala hospital in Dar es Salaam, Tanzania. BMC Heal Serv Res. 2014; 14:400.

19. Chakravarty A. Evaluation of service quality of hospital outpatient department services. Med J Armed Forces India. 2011;67(3):221-4.

20. Al Fraihi KJ, Latif SA. Evaluation of outpatient service quality in Eastern Saudi Arabia: Patient's expectations and perceptions. Saudi Med J. 2016;37(4):420-8. 
21. Mohd Suki N, Chwee Lian J, Suki NM. A comparison of human elements and nonhuman elements in private health care settings: Customers' perceptions and expectations. J Hosp Mark Public Relations. 2009;19(2):113-28.

22. Kalaja R, Myshketa R, Scalera F. Service quality assessment in health care sector: the case of Durres Public Hospital. Procedia - Soc Behav Sci. 2016;235(oct.):557-65.

23. Fatima I, Humayun A, Anwar MI, Iftikhar A, Aslam M, Shafiq M. How do patients perceive and expect quality of surgery, diagnostics, and emergency services in tertiary care hospitals? An evidence of gap analysis from Pakistan. Oman Med J. 2017;32(4):297-305.

24. Borges J, Carvalho S, Silva M. Quality of service provided to heart surgery patients of the Unified Health System-SUS. Rev Bras Cir Cardiovasc. 2010;25(2):172-82.

25. Regaira E, Sola M, Goñi R, Del Barrio M, Margall MA, Asiain MC. La calidad asistencial en cuidados intensivos evaluada por los pacientes mediante la escala Servqual. Enferm Intensiva. 2010;21(1):3-10.

26. Ghahramanian A, Rezaei T, Abdullahzadeh F, Sheikhalipour Z, Dianat I. Quality of healthcare services and its relationship with patient safety culture and nurse-physician professional communication. Heal Promot Perspect. 2017;7(3):168-74.

27. Mohebifar R, Hasani H, Barikani A, Rafiei S. Evaluating service quality from patients' perceptions: application of importance-performance analysis method. Osong Public Heal Res Perspect. 2016;7(4):233-8.

28. Manulik S, Rosińczuk J, Karniej P. Evaluation of health care service quality in Poland with the use of Servqual method at the specialist ambulatory health
29. Teshnizi SH, Aghamolaei T, Kahnouji K, Teshnizi SM, Ghani J. Assessing quality of health services with the Servqual model in Iran. A systematic review and meta-analysis. Int J Qual Heal Care. 2018;30(2):82-9.

30. Numpaque A, Rocha A. Modelos Servqual y Servqhos para la evaluación de calidad de los servicios de salud. Rev la Fac Med. 2016;64(4):715.

31. Parasuraman A, Berry LL, Zeithaml V. Refinement and reassessment of the Serqual scale. Journal of Retailing. 1991;67:420-50.

32. Babakus E, Mangold WG. Adapting the Servqual scale to hospital services: an empirical investigation. Health Serv Res. 1992; 26(6):767-86.

33. Rocha LR, Veiga DF, Oliveira PR, Song EH, Ferreira LM. Health service quality scale: Brazilian Portuguese translation, reliability and validity. BMC Health Serv Res. 2013;13(1):1.

34. Calixto M, Okino N, Hayashida M, Costa I, Trevizan M, Godoy S. Escala Servqual; validación en población mexicana. Texto Context Enferm. 2011;20(3):326-33.

35. Cabello E, Chirinos JL. Validación y aplicabilidad de encuestas Serviqual modificadas para medir la satisfacción de usuarios externos en servicios de salud. Rev Med Hered. 2012;23(2):88-95.

36. Moher D, Liberati A, Tetzlaff J, Altman DG. Preferred reporting items for systematic reviews and meta-analyses: The PRISMA statement. Int J Surg. 2010;8(5):336-41.

37. Vandenbroucke JP, Von Elm E, Altman DG, Gøtzsche PC, Mulrow $C D$, Pocock SJ, et al. Strengthening the reporting of observational studies in epidemiology (STROBE): Explanation and elaboration. PLoS Med. 2007;4(10):1628-54. 
38. Huang $Y$, Li SJ. Understanding quality perception gaps among executives, frontline employees, and patients: the outpatient services in Taiwan hospitals. Qual Manag Health Care. 2010;19(2):173-84.

39. Purcărea V, Gheorghe I, Petrescu $C$. The assessment of perceived service quality of public health care services in Romania using the Servqual scale. Procedia Economics and Finance. 2013;6:573-85.

40. Díaz IM, Villegas Periñán M. La calidad asistencial: concepto y medida. Dir Organ y Adm Empres. 2003;29:50-8.

41. Almaspoor $H$, Jannati $A$, Imani $A$, Salimlar S, Derakhshani N, Raef B. Comparing the health care system of Iran with various countries. Heal Scope. 2016; Inpress(Inpress):4-9.

42. Heshmati B, Joulaei H. Iran's health-care system in transition. Lancet. 2016;387(10013):29-30.

43. JMAJ. International medical community health system in Iran Ramin Mehrdad* Iranian Health System Outlook. JMAJ. 2009;52(521):69-73.

44. Silva MP, Duarte JM, Nara dos Santos AS, Tirones AL. Assessment of satisfaction among surgical clients of teaching hospitals : analysis of tangibility*. Cogitare Enferm. 2017;22(2).

45. Vissers J, Beech R. Health Operations Management: Patient flow logistics in health care. London: Taylor \& Francis; 2005.

46. Mahdavi M, Parsaeian M, Jaafaripooyan E, Ghaffari S. Recent Iranian health system reform: an operational perspective to improve health services quality. Int J Heal Policy Manag. 2017;7(1):70-4.

47. Dias OV, Vieira MA, Dias JP, Ramos LH. The dimensions of user satisfaction of the family health program: Trust and empathy. ACTA Paul Enferm. 2011;24(2):225-31.

48. Li M, Lowrie DB, Huang CY, Lu XC, Zhu YC, Wu XH, et al. Evaluating patients' perception of service quality at hospitals in nine Chinese cities by use of the ServQual scale. Asian Pac J Trop Biomed. 2015;5(6):497-504.

49. Jain SK, Gupta G. Measuring Service Quality: Servqual vs. Servperf Scales. Vikalpa. 2004;29(2):25-38.

50. Cronin JJ, Taylor SA. Servperf versus Servqual: Reconciling performance-based and perceptions-minus-expectations measurement of service quality. J Mark. 1994;58(1):125-31. 